\title{
Optical Lattices, Ultracold Atoms and Quantum Information Processing
}

\author{
D. Jaksch
}

October 24, 2018

\begin{abstract}
We review novel methods to investigate, control and manipulate neutral atoms in optical lattices. These setups allow unprecedented quantum control over large numbers of atoms and thus are very promising for applications in quantum information processing. After introducing optical lattices we discuss the superfluid (SF) and Mott insulating (MI) states of neutral atoms trapped in such lattices and investigate the SF-MI transition as recently observed experimentally. In the second part of the paper we give an overview of proposals for quantum information processing and show different ways to entangle the trapped atoms, in particular the usage of cold collisions and Rydberg atoms. Finally, we also briefly discuss the implementation of quantum simulators, entanglement enhanced atom interferometers, and ideas for robust quantum memory in optical lattices.
\end{abstract}

\section{Introduction}

The advent of experimental creation of Bose-Einstein condensates (BEC) [1] in dilute weakly interacting gases of bosonic Alkali atoms in 1995 has brought new and exciting prospects to the fields of atomic physics and quantum state engineering. All the atoms in a BEC show identical quantum properties and in many respects a BEC therefore behaves like one 'big' quantum particle. It also can be imaged without being destroyed, its interactions with laser light are much stronger than that of a single particle and a BEC is less fragile than one might expect. Experimentally observed life times are of the order of several seconds; BEC's can be moved, shaken and rotated without immediately destroying their striking quantum features. Therefore they turned out to be an ideal testing bed for fundamental quantum physics, basic techniques of quantum state engineering and for investigating atomic properties 2. The successful achievement of BEC and the early studies of their properties led to to the Nobel Prize in 2001 for the pioneers in this field E.A. Cornell, W. Ketterle, and C.E. Wieman [3]. 


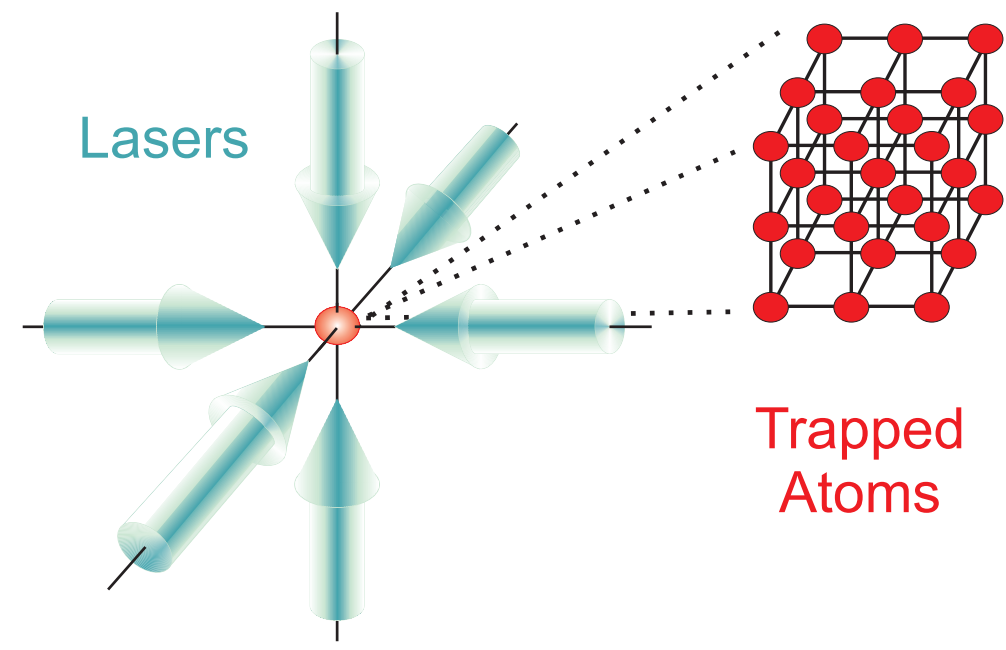

Figure 1: Laser setup and resulting optical lattice configuration in 3D.

The properties of a BEC described above make it an ideal tool for cold atom physics and quantum state engineering by quantum optical methods. One of the most promising experiments for future applications is the loading of a BEC into an optical lattice [4, 5, 6. Optical lattices are periodic conservative trapping potentials which are created by interference of travelling laser beams yielding standing laser waves in each direction (see Fig. 11). The laser light induces an AC-Stark shifts in atoms and thus acts as a conservative periodic potential. The usage of a BEC for loading has the advantage that its large atom density allows a filling of few particles per site $n \gtrsim 1$ while laser cooled atoms loaded into an optical lattice typically only allow a filling factor smaller than one. Furthermore the atoms loaded from a BEC are ultracold at temperatures very close to zero so that they practically behave as if their temperature was $T=0$, in particular all of them occupy the lowest Bloch band. The basic setup of optical lattices and the loading of a BEC will be described in detail in Sec. 2

An important novel feature which comes about because of the large filling factor $n \gtrsim 1$ is that interatomic interactions - due to $s$-wave collisions - of two or more atoms occupying the same lattice site become important. The interatomic interaction potential, which usually leads to incoherent collisions in a thermal cloud of atoms and is responsible for the mean field in a BEC, causes a coherent energy shift $U$ of two particles occupying a single lattice site. For the most frequently used alkali atoms ${ }^{87} \mathrm{Rb}$ and ${ }^{23} \mathrm{Na}$ the interaction is repulsive $U>0$ and thus the interaction energy competes with the kinetic energy $J$ gained by a particle when hopping from one lattice site to the next. Both of these parameters depend on the laser intensity. When increasing the laser intensity the barriers between neighbouring sites increases in height and 
$J$ goes down. At the same time two particles sitting in one site are pushed together closer resulting in an increased interaction energy. Therefore the competition between kinetic and interaction energy can be controlled by the laser intensity which is an external easily changeable control parameter. In Sec. 3 we will describe the physical consequences of different ratios $J / U$ and investigate the properties of the resulting ground states.

It turns out that the nature of the resulting quantum ground states allows for a number of applications ranging from the controlled creation of molecules [7, the study of strongly correlated quantum states [6], enhanced atom interferometry experiments and precision measurements [27, controlled entanglement creation, to quantum computing [8, 9, 10, 12, 13. In addition to having well defined initial motional states a number of different internal (hyperfine) atomic states can be trapped which results in additional well controlled degrees of freedom. Optical lattices are very versatile, easily altered by changing the laser setup and the control over the quantum state of the atoms by quantum optical techniques is unprecedented. Furthermore, decoherence times in optical lattices are large compared to typical experimental times which allows the study of Hamiltonian dynamics of strongly correlated quantum states with time dependent parameters and enables quantum information processing. All of these features make optical lattices a unique system to study. We will discuss some of the possible applications in Sec. 4 focussing on applications in quantum information processing. We will show how to implement quantum memory and quantum gates in optical lattices and review ideas for using optical lattices as a quantum simulator for complex strongly correlated systems.

The high degree of precision that can be obtained in manipulating quantum optical systems and the large degree of isolation from the environment has led to a number of different quantum optical setups which might be suitable for quantum computing. Several experiments in the groups of D. Wineland [15] and R. Blatt [17] on ion traps have convincingly demonstrated two qubit gates. The next major step in these efforts is to scale them to a larger number of ions which will probably be on the order of $\approx 30$ [16]. Also, photons in an optical cavity might be used to implement a quantum computer [14]. The biggest advantage of cavity systems is that they might allow to couple resting qubits (atoms) to flying qubits (photons) and thus enable the implementation of a quantum network.

There are also several non-quantum optical systems that have been identified as candidates for implementing a quantum computer. At the moment the most advanced system is NMR (Nuclear Magnetic Resonance) [18. However, although NMR systems can be used to perform demonstrations of quantum computing algorithms on very small systems it is as yet not known how to scale an NMR quantum computer. Other promising candidates are superconducting Josephson junction arrays [19], quantum dots [20] and somewhat more exotic systems like e.g. ammonia molecules con- 


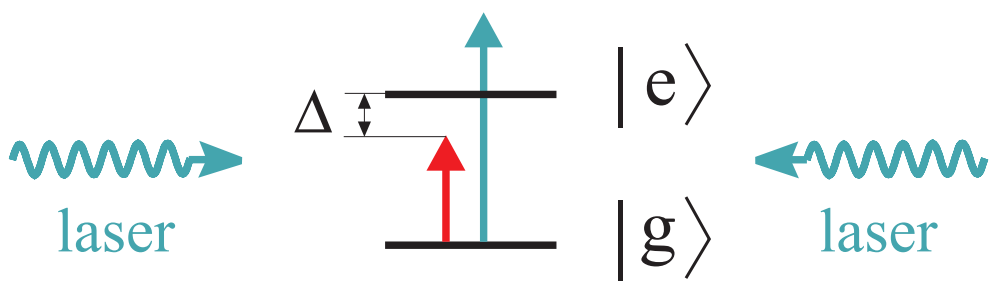

Figure 2: Interaction of a laser with a single atom. The red (blue) arrows indicate red (blue) detuned lasers.

fined inside fullerenes [21. Which of these setups will eventually lead to a successful implementation remains to be seen. However, as we will show for optical lattice setups in the remainder of this paper a lot of interesting quantum physics is contained in the above systems. Therefore, even if some of them will not succeed in performing scalable quantum computations, it is certainly worth investigating them to learn more about their astonishing quantum features.

\section{Loading a BEC into an optical lattice}

In this section we first briefly describe the creation of an optical lattice; specifically we show how the interaction between atoms and a laser field can lead to a conservative trapping potential. Then we investigate how such a lattice can be used to trap and manipulate neutral atoms from a BEC. We will also mention some of the complications and imperfections that arise in this context but not deal with them in detail.

\subsection{Optical lattices}

Let us for simplicity consider a two-level atom with an internal (hyperfine) ground state $|g\rangle$ and an excited state $|e\rangle$ in one spatial dimension only coupled to a monochromatic classical laser field with a detuning $\Delta$ as schematically shown in Fig. 2 The laser is assumed not to cause significant population in the excited state $|e\rangle$ and therefore we may neglect spontaneous emission from it. In a frame rotating with the laser frequency the Hamiltonian (with $\hbar=1$ ) for the atom is given by

$$
H_{\text {atom }}=\frac{\hat{p}^{2}}{2 m}+\Delta|e\rangle\langle e|-\frac{\Omega(\hat{x})}{2}(|e\rangle\langle g|+| g\rangle\langle e|) .
$$

Here $\hat{x}$ and $\hat{p}$ are the coordinate and momentum operators respectively, and the mass of the atom is $m$. The laser drives the transition between the two atomic states with a Rabi frequency $\Omega$ which is proportional to the laser field and the dipole transition matrix element of the atom. We now 
write the atomic wave function as $|\Psi(t)\rangle=\left|\Psi_{e}(t)\right\rangle|e\rangle+\left|\Psi_{g}(t)\right\rangle|g\rangle$ where $\left|\Psi_{e(g)}(t)\right\rangle=\langle e(g) \mid \Psi(t)\rangle$ and derive the equations of motion for the wave functions $\left|\Psi_{e}(t)\right\rangle$ and $\left|\Psi_{g}(t)\right\rangle$. We find

$$
\begin{aligned}
i \frac{d\left|\Psi_{g}(t)\right\rangle}{d t} & =\frac{\hat{p}^{2}}{2 m}\left|\Psi_{g}(t)\right\rangle-\frac{\Omega(\hat{x})}{2}\left|\Psi_{e}(t)\right\rangle, \\
i \frac{d\left|\Psi_{e}(t)\right\rangle}{d t} & =\left(\frac{\hat{p}^{2}}{2 m}+\Delta\right)\left|\Psi_{e}(t)\right\rangle-\frac{\Omega(\hat{x})}{2}\left|\Psi_{g}(t)\right\rangle .
\end{aligned}
$$

If the laser is far detuned, i.e. $|\Delta| \gg|\Omega|$ it is permissible to adiabatically eliminate the excited internal state of the atom $|e\rangle$. The adiabatic elimination consists in assuming that the wave function $\left|\Psi_{e}(t)\right\rangle$ adiabatically follows any change in the ground state wave function $\left|\Psi_{g}(t)\right\rangle$. We can thus set the left hand side of Eq. (3) equal to zero. Furthermore we assume the kinetic energy of an excited atom (and thus also the recoil energy $E_{R}=k^{2} / 2 m$ where $k=2 \pi / \lambda$ with $\lambda$ the laser wave length) to be much smaller than the detuning $E_{R} \ll|\Delta|$ and neglect it in Eq. (3) compared to $\Delta$. The equation Eq. (3) is now easily solvable and substituting its solution into Eq. (2) we find that the atom now moves according to the Schrödinger equation

$$
i \frac{d\left|\Psi_{g}(t)\right\rangle}{d t}=\left(\frac{\hat{p}^{2}}{2 m}+V_{0}(\hat{x})\right)\left|\Psi_{g}(t)\right\rangle
$$

where we have defined the optical potential as

$$
V_{0}(x)=-\frac{\Omega(x)^{2}}{4 \Delta} .
$$

We will always assume the atom to be interacting with a standing light wave which arises from the interference of two counter propagating laser beams. This means that the spatial dependence of the Rabi frequency is given by

$$
\Omega(x)=\Omega_{0} \sin (k x)
$$

where $\Omega_{0}$ is a constant depending on the laser intensity and the properties of the atom. We therefore find that the motion of the atom is effectively described by a periodic conservative potential with period $a=\lambda / 2$ and a depth controlled by the laser intensity. For a sufficiently deep lattice the atoms can be trapped near the potential minima which are also called lattice sites. In the vicinity of each lattice site the trap potential is well approximated by a harmonic oscillator potential with frequency

$$
\omega_{T}=\left|\frac{\Omega_{0} k}{\sqrt{2 \Delta m}}\right| .
$$




\subsection{Imperfections}

Above we have totally neglected spontaneous emission from the excited atomic level. We give an estimate of this effect by assuming that the atom is localized close to the minima of the optical lattice potential. For blue detuned optical lattices $(\Delta<0)$ the lattice sites are at positions of zero laser intensity while for a red detuned optical lattice $(\Delta>0)$ they are at locations of maximum laser intensity. Therefore in a blue lattice the effects of spontaneous emission on trapped particles are less strong than for red lattices. In a blue lattice we find the effective spontaneous emission rate $\gamma_{\text {eff }}^{b}$ of a trapped atom

$$
\gamma_{\mathrm{eff}}^{b} \approx-\frac{\gamma}{4 \Delta} \omega_{T}
$$

where $\gamma$ is the spontaneous emission rate from the excited atomic level. Therefore spontaneous emission will be negligible for large detunings $\Delta$. Indeed, it has been shown in an experiment by Friebel et al. 22] that the spontaneous emission rate in far detuned optical lattices can be of the order of several minutes.

Since we are interested in having a few atoms per lattice we have to consider the effects of light induced inelastic collisions between two atoms. In Fig. 3] we have schematically shown the interaction potentials for the different internal states $(S+S)$ and $(P+S)$ of two atoms approaching each other at a distance $r$. As a consequence of this interaction the detuning $\Delta$ of the laser field changes as the particles come close to each other and becomes $\Delta=0$ at the so called Condon point $R_{C}$. The probability for the two particles to be excited to the states $P+S$ increases around $R_{C}$. The atoms can then either form a quasistable molecule (for red detuning) which decays by spontaneously emitting a photon or they can be promoted to an unbound $S+P$ state with a large kinetic energy equal to the detuning of the laser at $r=\infty$. Both of these effects should be kept as small as possible to minimize loss from the optical lattice. There are several ways to do so:

- Avoid a Condon point by detuning far to the red. However, this will lead to a larger spontaneous emission rate compared to the case of a blue lattice.

- Avoid a red detuning that puts the Condon point close to a quasi bound molecular state (shown in Fig. 3) as this enhances the probability of creating such molecules.

- Choose the detuning such that the two potential curves have the largest possible angle with each other at the Condon point. This makes the probability of finding the two particles in the region where $\Delta \approx 0$ small.

The above conditions for neglecting spontaneous emission and making laser induced collisions unimportant can be fulfilled quite well experimentally and 


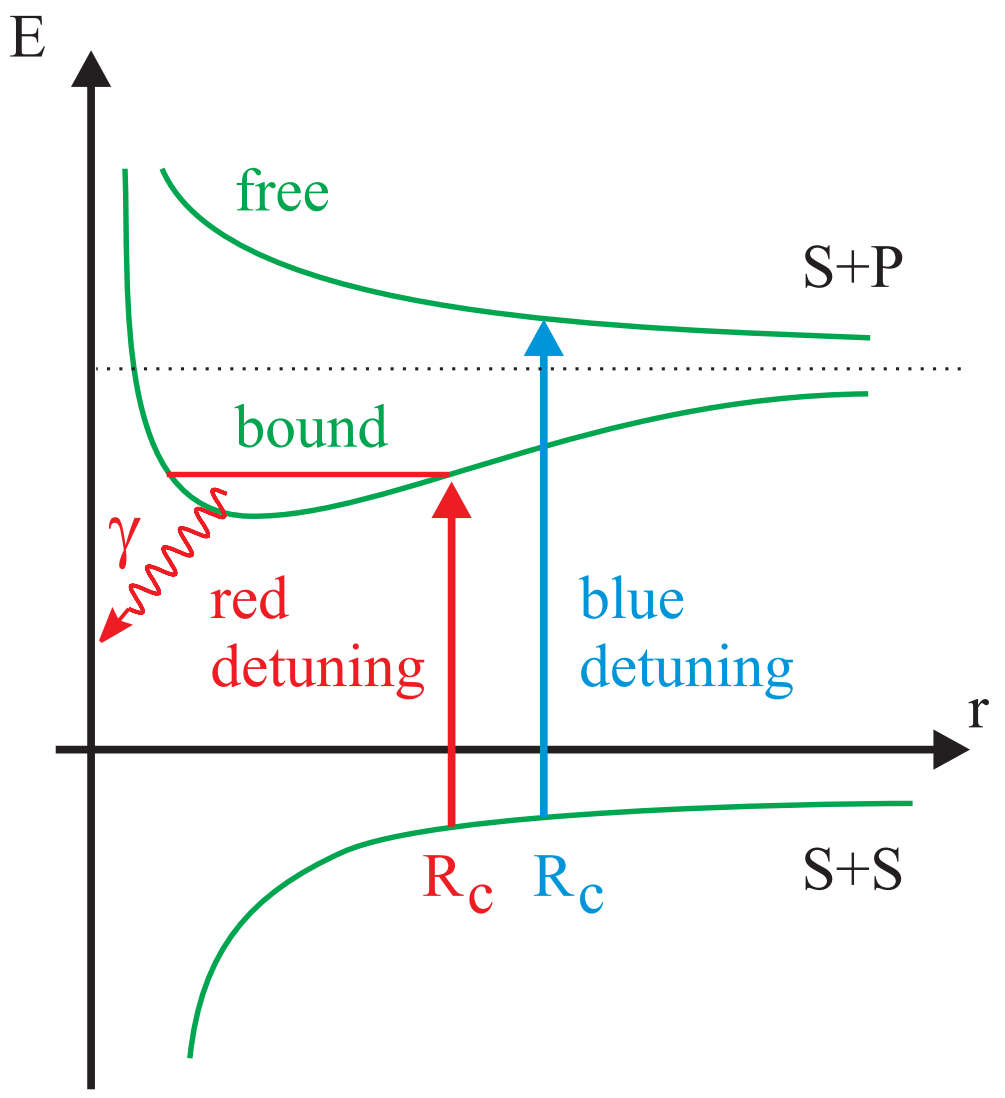

Figure 3: Schematic interaction potential curves for two atoms at distance $r$. The spontaneous emission from a bound molecular state $\gamma$ is also indicated.

then our above simple model for an optical lattice as a conservative potential is valid.

\subsection{Experimental setups}

The situation where one pair of counter propagating laser beams interferes and yields a trap potential in $1 \mathrm{D}$ while the atoms are only weakly trapped by a magnetic field (or not trapped at all) in the other two dimensions is usually called a 1D optical lattice. A BEC was first loaded into a 1D lattice by B.P. Anderson and M.A. Kasevich [4]. In this experiment a magnetically trapped BEC is loaded into a weak vertical optical lattice while at the same time turning off the magnetic trapping almost completely. The experiment is designed such that the interplay between the gravitational force and the optical lattice leads to a peculiar interference pattern of atoms falling out of the lattice which allows to study the coherence properties of the original BEC. 


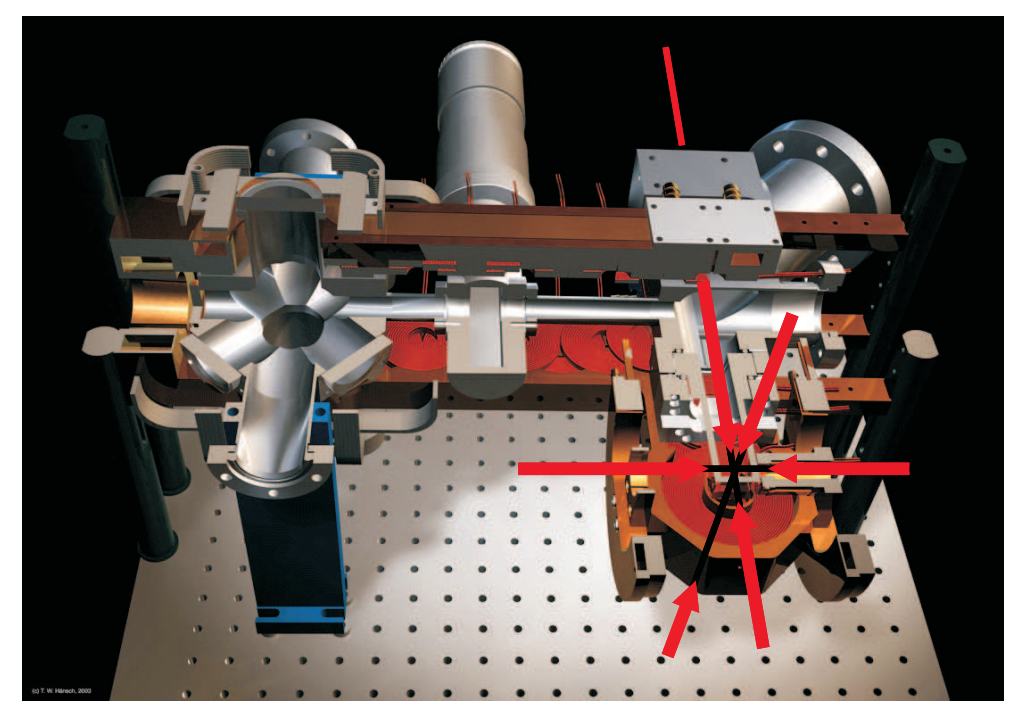

Figure 4: Experimental setup used in Munich to load a BEC into a 3D optical lattice [6. The BEC is produced in a magnetic trap located at the left hand side of the figure and is then transported to the right where optical access from all directions is possible as shown by the red arrows.

A 1D optical lattice setup is theoretically easily extended to the 3D situation as shown in Fig. 1. Three pairs of counter propagating laser beams interfere to produce an optical potential which traps the atoms in each dimension. Experimentally, however, the achievement of this situation is less straightforward. The experimental setup has to allow for optical access to the BEC from three different orthogonal directions and this is usually incompatible with producing the magnetic trap required to create the BEC. For this reason the group headed by $\mathrm{T}$. Hänsch in Munich realized the concept of spatially separating the production of a BEC and performing the actual optical lattice experiment. Their setup is schematically shown in Fig. 4. A number of coils are used to create an inhomogeneous magnetic field that guides the BEC out of its original position and transports it into the 'science chamber' where optical access from three directions is possible and allows the realization of a 3D optical lattice. Next we will discuss the novel features of a BEC loaded into such a 3D optical lattice. In particular we will investigate the effects of interactions between the particles which are responsible for striking new features in comparison to previous experiments on optical lattices. 


\section{Superfluids and Mott insulators}

The above setup with 3D lattice geometry was predicted to yield a qualitatively new behaviour of a dense cloud of ultracold atoms in 1998 [23] and was experimentally realized in 2001 by M. Greiner et al. 6]. We can gain some insight into the properties of the atoms trapped in the lattice by looking at two limiting cases; the superfluid limit where the kinetic energy dominates the repulsive interaction and the opposite limit which is called the Mott insulating limit.

\subsection{Superfluid limit}

When the optical lattice is shallow the kinetic energy dominates over the repulsion of two particles sitting in the same lattice site. In this situation nearly all the atoms occupy the same single particle state. For very small interaction energies this single particle state is very close to the Bloch wave function with quasi momentum $q=0$ of the lowest Bloch band, i.e. the single particle ground state of the periodic potential. As this wave function is delocalized over the system there is long range off-diagonal coherence over the whole lattice. States like this are very well described by the Gross-Pitaevskii equation 2] which takes the interaction into account via a simple mean field approximation. Neglecting interactions altogether we can understand the form of the underlying Hamiltonian intuitively. We define a bosonic destruction operator $a_{l}$ destroying an atom in the lattice site labelled $l$. Its adjoint operator $a_{l}^{\dagger}$ correspondingly creates a particle in this lattice site and $n_{l}=a_{l}^{\dagger} a_{l}$ counts the number of particles in site $l$. By hopping (tunneling) from one lattice site to an adjacent site an atom loses a kinetic energy $J$ depending on the height of the barrier between the sites and the Hamiltonian therefore reads

$$
H_{\mathrm{SF}}=-J \sum_{\langle l, m\rangle} a_{l}^{\dagger} a_{m}
$$

where the sum runs over all pairs of nearest neighbours in the lattice. An atom in the lattice will therefore minimize its energy when it is in a superposition state of many lattice sites.

A more detailed investigation shows that the state

$$
\left|\Psi_{\mathrm{SF}}\right\rangle \propto\left(\sum_{l} a_{l}^{\dagger}\right)^{N}|\mathrm{vac}\rangle
$$

of $N$ particles with $|\mathrm{vac}\rangle$ the vacuum minimizes the energy. This state clearly corresponds to each particle occupying the lowest Bloch state and spreads the wave function of each particle over the whole lattice as indicated in Fig. 5. The state therefore shows large off diagonal long range coherence as can be seen by correlating the destruction of an atom in lattice site $l$ with 

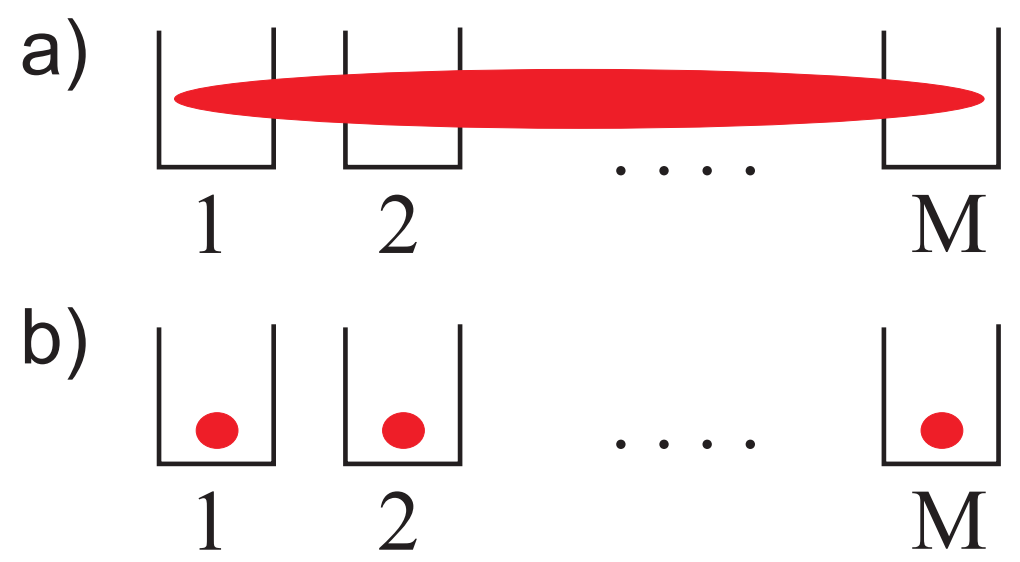

Figure 5: Schematic representation of a) a SF state and b) a MI state with commensurate filling $n=1$ in an optical lattice with $M$ sites.

the creation of a particle in site $m$. The corresponding correlation function is found by the expectation value $\rho_{l, m}=\left\langle a_{l}^{\dagger} a_{m}\right\rangle$. In the SF regime this correlation function is independent of the distance between sites $l$ and $m$ and its modulus is given by $\left|\rho_{l, m}\right|=N / M$ where $M$ is the total number of lattice sites. We also note that the particle number fluctuations $\left(\Delta n_{l}\right)^{2}=N / M$ in this state are large.

The lowest lying excitations in the SF limit are determined by the Bloch band and consist of waves with quasi momentum $q$. The energy of the first excited state scales like $\epsilon_{1} \propto J / M^{2}$ which tends to 0 for large systems $M \rightarrow \infty$. This vanishing gap between the ground state and low lying excited states makes the SF state rather fragile and seems to render it very difficult to adiabatically load a BEC into an optical lattice. Fortunately, however, the turning on of a lattice does not induce quasi-momenta and therefore does not excite low lying excitations. Therefore loading a lattice from a BEC is possible at reasonable speed without significant heating of the atomic cloud.

\subsection{Mott insulating limit}

By increasing the lattice depth the kinetic energy goes down because the barrier height increases. At the same time the size of each lattice site decreases which causes the atoms to repel each other more strongly. Again we can understand this situation intuitively. We neglect any kinetic energy and only consider the interaction energy of two particles in the same lattice site. As usual the two particle interaction energy increases with the square of the particle density which itself is proportional to the square of the number of 
particles per well. The Hamiltonian therefore is given by

$$
H_{\mathrm{MI}}=\frac{U}{2} \sum_{l} n_{l}\left(n_{l}-1\right) .
$$

Here the parameter $U$ is the interaction energy of two particles in the same lattice site. Note the -1 term which is usually neglected in most mean field theories. It ensures that a single particle does not gain any interaction energy.

In this situation particles in the optical lattice minimize their energy by staying away from each other instead of spreading out over the whole lattice. Let us consider the case of commensurate filling $M=N$ where the smallest interaction energy is obtained by putting each particle into its individual lattice site. A state like this is written as

$$
\left|\Psi_{\mathrm{MI}}\right\rangle \propto \prod_{l} a_{l}^{\dagger}|\mathrm{vac}\rangle
$$

is called a Mott insulator, and schematically shown in Fig. 5b. It does not have any interaction energy $\left\langle\Psi_{\mathrm{MI}}\left|H_{\mathrm{int}}\right| \Psi_{\mathrm{MI}}\right\rangle=0$ while the above SF state would have an interaction energy of $\left\langle\Psi_{\mathrm{SF}}\left|H_{\mathrm{int}}\right| \Psi_{\mathrm{SF}}\right\rangle=U N(N-1) / 2 M^{2}$. Furthermore, the MI state has no long range coherence $\rho_{l, m}=0$ for $l \neq m$ and also the particle number fluctuations are zero $\Delta n_{l}^{2}=0$.

The MI state is much more stable than the SF state. The lowest lying excitations are obtained by taking one particle out of its site and putting it on top of an atom sitting in another lattice site. The energy required for this is $U$ independent of the size $M$, i.e. the system is characterized by a finite gap $U$ large compared to the small excitation energies in the SF limit.

Experimentally the situation of commensurate filling can be achieved by suitably superimposing the optical lattice with a much weaker magnetic trapping potential. The atoms fill those lattice sites which are in the center of the wide trap and avoid the outer ones so that we obtain commensurate filling in the center of the lattice. The magnetic trap acts as a 'soft' boundary which adjusts to the number of particles. Note, however, that the nature of the MI will be altered close to this boundary.

\subsection{Intermediate regime}

If the two parameters $J$ and $U$ are of similar magnitude the situation is more complicated. Kinetic energy and interactions compete with each other and only a detailed investigation shows whether the system is a SF or a MI. For commensurate filling of the lattice with a filling factor of $n=1$ one easily finds that the interaction wins for $U>U_{c}$ while the kinetic energy dominates for $U<U_{c}$, where the critical point is $U_{c} \approx 5.8 z J$. Here $z=2 d$ is the number of nearest neighbours in a $d$-dimensional lattice. For larger 


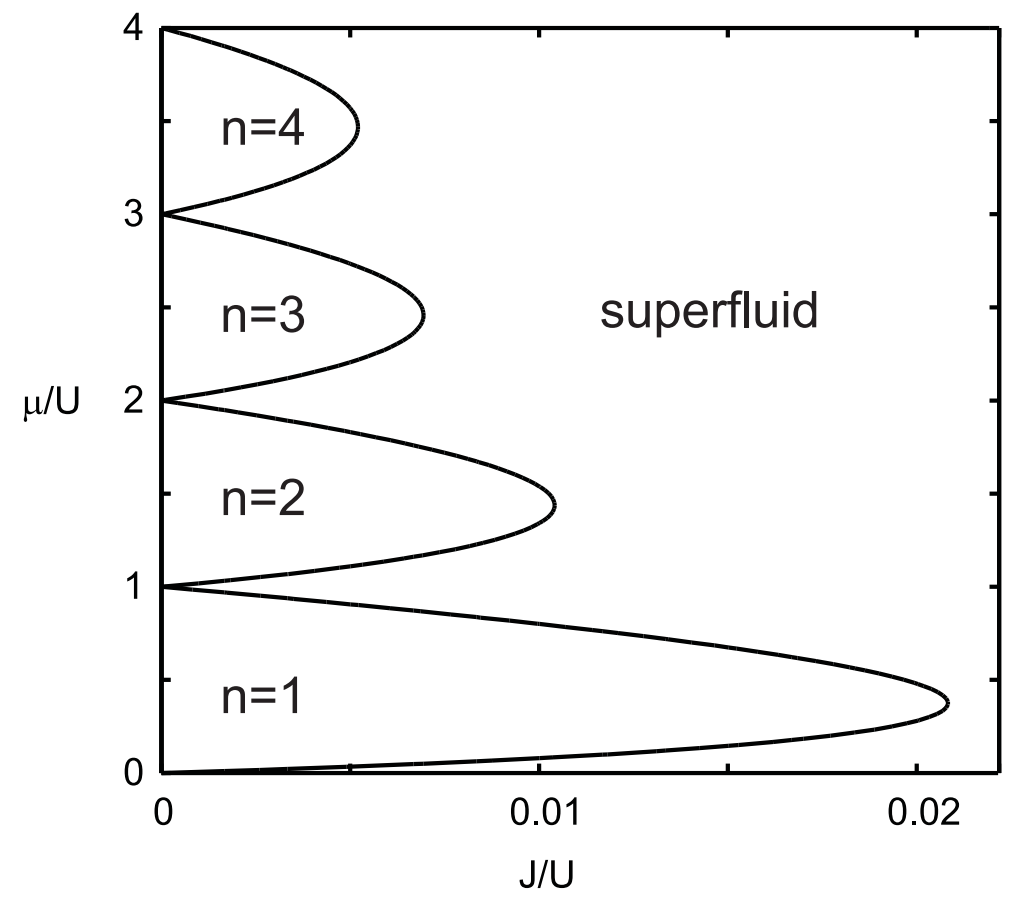

Figure 6: Phase diagram of the 3D Bose-Hubbard model showing the MI regions with filling factor $n$ and the $\mathrm{SF}$ region for different ratios $J / U$ and chemical potentials $\mu / U$. Note that the simple mean field approximation used here does not yield $U_{c}=5.8 z J$.

commensurate fillings of the lattice the value of $U_{c}$ increases because the interaction energy goes up quadratically with the filling while the gained kinetic energy only increases linearly with the filling factor.

For incommensurate fillings the system is best described in the grand canonical ensemble where the mean particle number is fixed by a particle reservoir of chemical potential $\mu$. By using simple mean field theory the $\mathrm{SF}$ and MI regions can be found approximately; the numerical result is shown in Fig. 6. When varying $J$ over the critical point the ground state abruptly changes its nature and the transition between the SF and the MI regime at temperature $T=0$ takes place in a so called quantum phase transition if the lattice is sufficiently large. We can understand the basic physics of a quantum phase transition by considering a Hamiltonian of the form $H=H_{1}+g H_{2}$ where the two parts of $H$ commute, i.e. $\left[H_{1}, H_{2}\right]=0$ and $g>0$ is a parameter. Since $H_{1}$ and $H_{2}$ commute the eigenstates of $H$ are independent of $g$ while the eigenvalues change with $g$. For small values of $g<g_{c}$ the ground state of $H$ will be determined by the ground state of $H_{1}$ while for large values of $g>g_{c}$ it is given by the ground state of $H_{2}$. If these two ground states have totally different properties the system alters its 

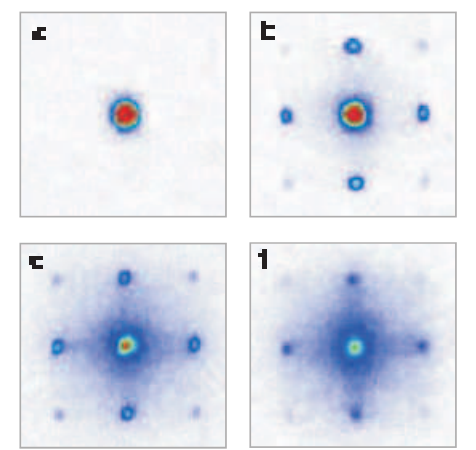

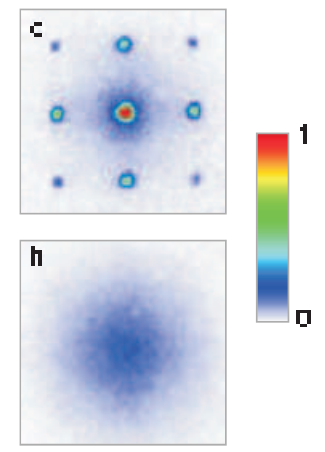

Figure 7: Interference patterns for decreasing $J / U$ (a-h). For $U<U_{c}$ the long range coherence leads to an interference pattern which clearly indicates the band structure $(\mathrm{a}-\mathrm{d})$. The interference fringes vanish when the system enters the MI regime $U>U_{c}(\mathrm{e}-\mathrm{h})$.

behaviour suddenly when the parameter $g$ is changed over the critical point $g_{c}$. A quantum phase transition also occurs when the commutator of the two parts of the Hamiltonian is not exactly zero but does not increase with the size of the system and therefore can be neglected in the case of large systems. This is exactly the situation we encounter in the so called BoseHubbard model with Hamiltonian $H_{\mathrm{BHM}}=H_{\mathrm{SF}}+H_{\mathrm{MI}}$ we are considering here for a changing ratio of $J / U$.

An optical lattice has the unique feature of permitting studies of the dynamics of the quantum phase transition in $H_{\mathrm{BHM}}$, i.e., the parameters $U$ and $J$ can be changed on a time scale much smaller than the coherence time of the system but still sufficiently slow to avoid significant excitations. The experiments carried out by M. Greiner et al. [6] to study this dynamics started out from a Bose-condensate in a magnetic trap. Then the optical lattice was (adiabatically) turned on. Its depth determines the ratio $J / U$ and thus the kind of ground state in the optical lattice. To measure the properties of the atomic state the lattice potential is then turned off and the particles are allowed to expand freely before their density distribution is measured. In the case of a SF state which has long range off diagonal coherence the particles interfere and the resulting interference pattern clearly indicates the band structure of the lattice. When the system is in a MI state there is no long range coherence and therefore the interference pattern disappears. This behaviour is similar to interference in optics experiments where interference patterns also vanish when there is lack of coherence. The corresponding interference patterns as measured in [6] are shown in Fig. 7 for different depths of the optical lattice.

Next we discuss some of the applications of MI states in optical lattices focussing on applications in quantum computing. Afterwards we look at 
atomic interferometry enhanced by using multi particle entangled states.

\section{Quantum information processing in optical lat- tices}

The experiments on the SF to MI transition are a very important step towards realizing theoretical ideas for controlled entanglement creation in optical lattices by interactions between neutral atoms [8, 10, 9, 12. Together with single particle manipulations such entanglement operations constitute the basic building blocks for a quantum computer 24] which fulfils all the basic requirements as set out by D. DiVincenzo [25]. First important experimental steps towards realizing multi particle entangled states have recently been achieved by Mandel et al. 26. In this section we will first discuss the requirements for implementing a quantum computer and then show how they can be satisfied in an optical lattice.

\subsection{What is quantum information processing?}

A computation can be considered as a physical process that transforms an input into an output. A classical computation is that in which the physical process is based on classical laws while the physical process in quantum computation is based on quantum laws and in particular on the superposition principle. In quantum information processing the inputs and outputs are encoded into the states of the system and the computation evolves the input state by a designed well controlled unitary time evolution. In general, if our algorithm consists of evaluating a given function $f$, we can design an interaction Hamiltonian such that the resulting unitary evolution transforms the input states according to $|j\rangle \otimes|0\rangle \rightarrow|j\rangle \otimes|f(j)\rangle(j=1, N)$.

Using a quantum computer we can do more than with a classical computer. When preparing the input state in a superposition of all $L=2^{N}$ basis states for $N$ qubits it will be transformed according to

$$
\frac{1}{\sqrt{L}} \sum_{\ell=1}^{L}|\ell\rangle \otimes|0\rangle \rightarrow \frac{1}{\sqrt{L}} \sum_{\ell=1}^{L}|\ell\rangle \otimes|f(\ell)\rangle
$$

in a single run. All the values of the function $f$ are present in this superposition. However, we do not have access to all of this information since after a measurement we will only obtain one result with a certain probability. The property of using quantum superpositions to run the computer only once was termed quantum parallelism by Feynman.

\subsubsection{Requirements}

A quantum computer consists of a quantum register (in our case an array of atoms in the optical lattice) that can be manipulated and measured in 
a controlled way. If we want to build a quantum computer, we need the following elements [25]:

(i) A set of qubits which are two-level systems forming the quantum register. In optical lattices this set of qubits is formed by two internal states of the atoms trapped in the lattice. We denote by $\left\{|0\rangle_{j},|1\rangle_{j}\right\}$ two orthogonal states of the $j$-th qubit (also called the computational basis), so that a state $|\Psi\rangle$ of all the qubits in the quantum register can be written as

$$
\begin{aligned}
|\Psi\rangle & =\sum_{j_{1}, j_{2}, \ldots j_{N}=0}^{1} c_{j_{1} j_{2} \ldots j_{N}}\left|j_{1}\right\rangle_{1} \otimes\left|j_{2}\right\rangle_{2} \otimes \ldots \otimes\left|j_{N}\right\rangle_{N} \\
& \equiv \sum_{j_{1}, j_{2}, \ldots j_{N}=0}^{1} c_{j_{1} j_{2} \ldots j_{N}}\left|j_{1}, j_{2}, \ldots j_{N}\right\rangle .
\end{aligned}
$$

These qubits can be in superposition and entangled states, which gives the extraordinary power to the quantum computer; they also have to be well isolated from the environment to prevent decoherence.

(ii) A universal set of quantum gates which allows the controlled manipulation of the qubits according to any unitary operation $U$ so that $|\Psi\rangle \rightarrow U|\Psi\rangle$. Fortunately, this task is enormously simplified given the fact that any $U$ can be decomposed as a product of gates belonging to a small set, a so-called universal set of gates, i.e. if we are able to perform the gates of this set we will be able to perform any unitary operation on the register by applying a sequence of them. There are many sets of universal gates and we will concentrate on the set containing one two qubit phase gate and single qubit operations as this is the one most naturally arising in optical lattice setups. Note that two-qubit gates require interactions between the qubits, and therefore are the more difficult ones in practice.

(iii) Detection of the output state. One should be able to measure each qubit in its computational basis. This process requires the interaction with a measurement apparatus in an irreversible way and is thus not governed by a unitary time evolution.

(iv) Initial state preparation. We must be able to erase quantum registers to prepare the initial quantum state, for example the state $|0,0, \ldots, 0\rangle$. This is actually not an extra requirement, it is enough to detect the qubits and to apply a single-qubit gate flipping the qubit $|1\rangle \rightarrow|0\rangle$ and $|0\rangle \rightarrow|1\rangle$ if necessary.

(v) Scalability of the system. The difficulty of performing gates, measurements, etc., should not grow (exponentially) with the number of qubits. Otherwise, the gain in the quantum algorithms would be lost.

(vi) In addition to the above we also require networking ability. The static qubits used to perform quantum computations should be transformable into flying qubits which can easily be transmitted between different locations. 
In the following we will discuss how to implement an initialized quantum register and a universal set of quantum gates in an optical lattice setup. Due to its periodic structure the optical lattice provides an intrinsically scalable setup. The problem of detecting the output state is closely related to addressing single qubits as required for performing single qubit operations.

A discussion of how to achieve networking ability lies beyond the scope of this paper.

\subsection{Quantum memory and single qubit gates in optical lat- tices}

In optical lattices two states of the ground state hyperfine manifold of the trapped atoms $|a\rangle \equiv|0\rangle$ and $|b\rangle \equiv|1\rangle$ are ideal candidates to implement a qubit. However, in order to be able to manipulate and control atomic qubits it is necessary to know the position of each of the atoms precisely. While random filling of optical lattices from laser cooled atoms and a superfluid filling of a lattice (with large particle number fluctuations as discussed above) do not provide sufficient knowledge on the position of the atoms a MI state is ideally suited for this purpose. All the lattice sites are filled, i.e. each lattice site contains a qubit and the fluctuations in the occupation numbers are very small. For an appropriate choice of internal atomic states the optical lattice allows to trap both of them and thus can hold a quantum register for storing quantum information.

In principle it is straightforward to induce single qubit gates by using Raman transitions between the two internal states $|a\rangle$ and $|b\rangle$. Raman transitions with Rabi frequency $\Omega_{R}$ and detuning $\delta$ are described by the Hamiltonian

$$
H_{R}=\frac{1}{2}\left(\Omega_{R}|a\rangle\langle b|+\text { h.c. }\right)+\delta|b\rangle\langle b|,
$$

which induce rotations of the qubit state on the Bloch sphere. The axis and angle of this rotation depend on the choice of laser parameters and can be chosen freely. The major problem in inducing single qubit operations is the addressing of a single atom as it is difficult to focus a laser to spots of order of an optical wave length which is the typical separation between atoms in the lattice. Possible solutions to this difficulty are using schemes for pattern loading [23, 30], where only specific lattice sites are filled with atoms (cf. Fig. 8) or using additional marker atoms which specify the atom the laser is supposed to interact with.

MI atoms in an optical lattice have already experimentally been used as qubits and it has been shown that they support multi particle entangled states [26]. The robustness of these qubits is, however, limited by stray magnetic fields and spin echo techniques need to be used to perform the experiments successfully. A different approach to obtaining robust quantum memory uses a more sophisticated encoding of the qubits [27]. A 1D chain 


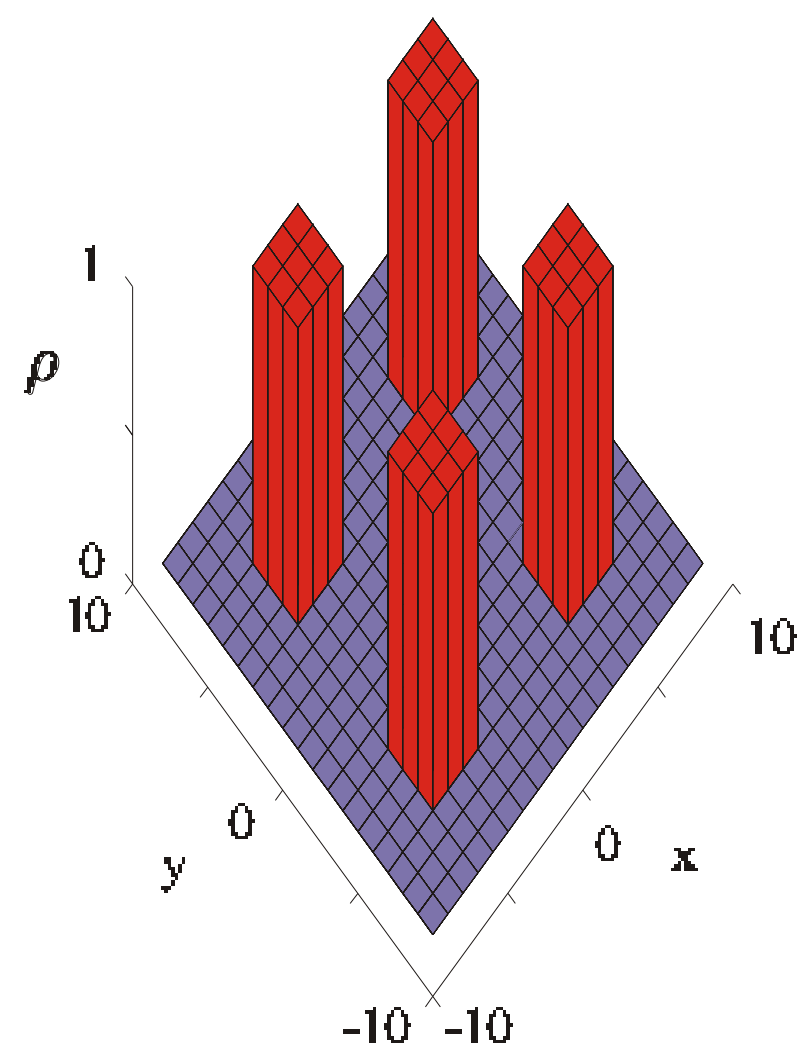

Figure 8: Selective filling of optical lattice sites in a superlattice structure. The red boxes indicate lattice sites filled with exactly one particle. The blue boxes show lattices sites that are empty.

with an even number of atoms encodes a single qubit in the states $|0\rangle=$ $|a b a b \cdots a b\rangle$ and $|1\rangle=|b a b a \cdots b a\rangle$. These states both contain the same number of atoms in internal states $|a\rangle$ and $|b\rangle$ and therefore interact with magnetic fields in exactly the same way avoiding dephasing of the quantum information stored in the chain. This and the fact that all the atoms have to flip their internal state to get from one logical state to the other make these qubits very robust against the most dominant experimental sources of decoherence. However, at the same time this robustness makes it more difficult to manipulate them. A laser pulse no longer corresponds to a simple rotation on the Bloch sphere spanned by the logical states $|0\rangle$ and $|1\rangle$ which makes the realization of a single qubit gate difficult. We will describe how these problems can be circumvented in Sec. 4.5 and further details can be found in [27]. 


\subsection{Two qubit operations}

Implementing a two qubit gate is more challenging than the single qubit gates. The different schemes for two qubit gates can be classified in two categories. The first version relies on the concept of a quantum data bus; the qubits are coupled to a collective auxiliary quantum mode, like e.g. a phonon mode in an ion trap, and entanglement is achieved by swapping the qubits to excitations of the collective mode. The second concept which is the basis for two qubit gates between atoms in optical lattices deploys controllable internal-state dependent two-body interactions. Examples for different interactions are coherent cold collisions of atoms, optical dipole-dipole interactions [8, 9] and the 'fast' two-qubit gate based on large permanent dipole interactions between laser excited Rydberg atoms in static electric fields [10]. Besides these dynamical schemes for entanglement creation it is also possible to generate entanglement by purely geometrical means [28. We will now discuss the different ways to achieve two qubit operations in optical lattices

\subsubsection{Entanglement via coherent ground state collisions}

The interaction terms introduced in Eq. (11) which describe $s$-wave collisions between ultracold atoms in one lattice site are analogous to Kerr nonlinearities between photons in quantum optics. For atoms stored in optical lattices these nonlinear atom-atom interactions can be large [8], even for interactions between individual pairs of atoms, thus providing the necessary ingredients to implement two-qubit gates.

We consider a situation where two atoms in a superposition of internal states $|a\rangle$ and $|b\rangle$ are trapped in the ground states of two optical lattice sites (see Fig. 9a). Initially, at time $t=-\tau$ these wells are centered at positions sufficiently far apart so that the particles do not interact. The optical lattice potential is then moved state selectively and for simplicity we assume that only the potential for a particle in internal state $|a\rangle$ moves to the right and drags along an atom in state $|a\rangle$ while a particle in state $|b\rangle$ remains at rest. Thus the wave function of each atom splits up in space according to the internal superposition of states $|a\rangle$ and $|b\rangle$. When the wave function of the left atom in state $|a\rangle$ reaches the second atom in state $|b\rangle$ as shown in Fig. 9b they will interact with each other. However, any other combination of internal states will not interact and therefore this collision is conditional on the internal state. A specific laser configuration achieving this state dependent atom transport has been analyzed in Ref. [8] for Alkali atoms, based on tuning the laser between the fine structure excited $p$-states. The trapping potentials can be moved by changing the laser parameters. Such trapping potentials could also be realized with magnetic and electric microtraps 29].

We therefore only need to consider the situation where atom 1 is in state 


\section{atom $1 \quad$ atom 2}

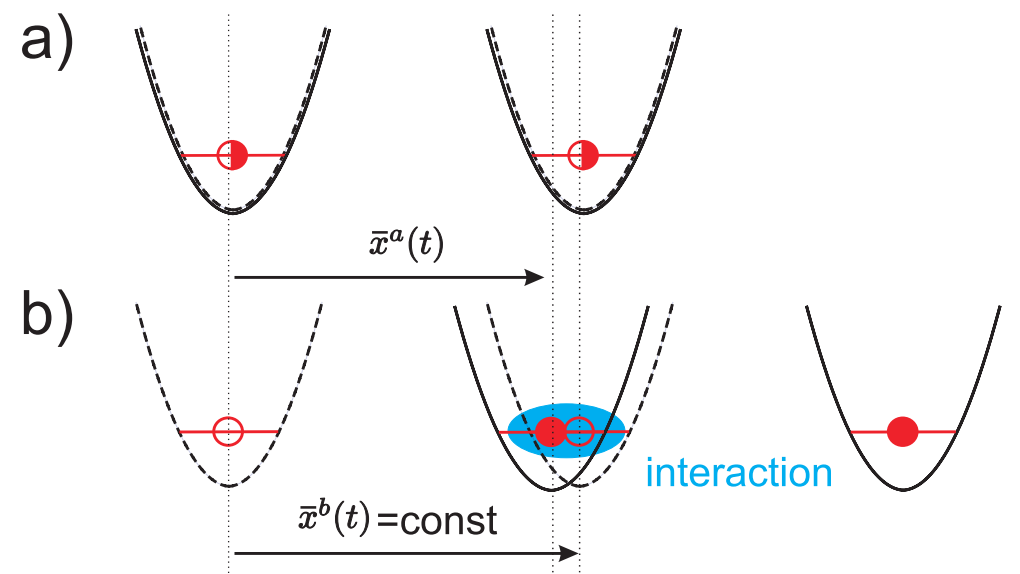

Figure 9: We collide one atom in internal state $|a\rangle$ (filled circle, potential indicated by solid curve) with a second atom in state $|b\rangle$ (open circle, potential indicated by dashed curve)). In the collision the wave function accumulates a phase according to Eq. (17). a) Configurations at times $t= \pm \tau$ and b) at time $t$.

$|a\rangle$ and particle 2 is in state $|b\rangle$ to analyze the interactions between the two atoms. The positions of the potentials are moved along trajectories $\bar{x}^{a}(t)$ and $\bar{x}^{b}(t)=$ const so that the wave packets of the atoms overlap for a certain time, until they are finally restored to the initial position at the final time $t=\tau$. This situation is described by the Hamiltonian

$$
H=\sum_{\beta=a, b}\left[\frac{\left(\hat{p}^{\beta}\right)^{2}}{2 m}+V^{\beta}\left(\hat{x}^{\beta}-\bar{x}^{\beta}(t)\right)\right]+u^{\mathrm{ab}}\left(\hat{x}^{a}-\hat{x}^{b}\right) .
$$

Here, $\hat{x}^{a, b}$ and $\hat{p}^{a, b}$ are position and momentum operators, $V^{a, b}\left(\hat{x}^{a, b}-\bar{x}^{a, b}(t)\right)$ describe the displaced trap potentials and $u^{\mathrm{ab}}$ is the atom-atom interaction term (which lead to the interaction term in Eq. (11)). Ideally, we want to implement the transformation from before to after the collision,

$$
\psi_{0}^{a}\left(x^{a}-\bar{x}^{a}(-\tau)\right) \psi_{0}^{b}\left(x^{b}-\bar{x}^{b}(-\tau)\right) \rightarrow e^{i \phi} \psi_{0}^{a}\left(x^{a}-\bar{x}^{a}(\tau)\right) \psi_{0}^{b}\left(x^{b}-\bar{x}^{b}(\tau)\right),
$$

where each atom remains in the ground state $\psi_{0}^{a, b}$ of its trapping potential and preserves its internal state. The phase $\phi=\phi^{a}+\phi^{b}+\phi^{\mathrm{ab}}$ will contain a contribution $\phi^{\mathrm{ab}}$ from the interaction (collision) and (trivial) single particle kinematic phases $\phi^{a}$ and $\phi^{b}$. The transformation Eq. (17) can be realized in the adiabatic limit, whereby we move the potentials slowly on the scale given by the trap frequency, so that the atoms remain in their motional 
ground state. In this case the collisional phase shift is given by $\phi^{\mathrm{ab}}=$ $\int_{-\infty}^{\infty} d t \Delta E(t) / \hbar$, where $\Delta E(t)$ is the energy shift induced by the atom-atom interactions

$$
\Delta E(t)=\frac{4 \pi a_{s} \hbar^{2}}{m} \int d x\left|\psi_{0}^{a}\left(x-\bar{x}^{a}(t)\right)\right|^{2}\left|\psi_{0}^{b}\left(x-\bar{x}^{b}(t)\right)\right|^{2},
$$

with $a_{s}$ the $s$-wave scattering length. In addition we assume that $|\Delta E(t)| \ll$ $\hbar \omega_{T}$ so that no sloshing motion is excited.

The interaction phase thus only applies when the atoms are in internal state $|a, b\rangle$ but not otherwise. Carrying out the above state selective collision with a phase $\phi^{\mathrm{ab}}=\pi$ we obtain (up to trivial phases) the mapping (as above we identify $|a\rangle \equiv|0\rangle$ and $|b\rangle \equiv|1\rangle)$

$$
\begin{aligned}
& |0,0\rangle \rightarrow|0,0\rangle \\
& |0,1\rangle \rightarrow-|0,1\rangle \\
& |1,0\rangle \rightarrow|1,0\rangle \\
& |1,1\rangle \rightarrow|1,1\rangle
\end{aligned}
$$

which realizes a two-qubit phase gate that is universal in combination with single-qubit rotations.

\subsubsection{State-selective interaction potential}

An alternative possibility, for a nontrivial logical phase to be obtained, is to rely on a state-independent trapping potential, while defining a procedure where different logical states couple to each other with different energies. An example is given by the interaction between state-selectively switched electrical dipoles [10].

In each qubit, the hyperfine ground states $|a\rangle \equiv|0\rangle$ is coupled by a laser to a given Stark eigenstate $|r\rangle$ which does not correspond to a logical state. The internal dynamics is described by a model Hamiltonian

$$
\begin{aligned}
H_{I}\left(t, \mathbf{x}_{1}, \mathbf{x}_{2}\right)= & \sum_{j=1,2}\left[\delta_{j}(t)|r\rangle_{j}\langle r|-\frac{\Omega_{j}\left(t, \mathbf{x}_{j}\right)}{2}\left(|a\rangle_{j}\langle r|+\text { h.c. }\right)\right] \\
& +u|r\rangle_{1}\langle r|\otimes| r\rangle_{2}\langle r|
\end{aligned}
$$

with $\Omega_{j}\left(t, \mathbf{x}_{j}\right)$ Rabi frequencies, and $\delta_{j}(t)$ detunings of the exciting lasers. Here, $u$ is the dipole-dipole interaction energy between the two particles. We have neglected any loss from the excited states $|r\rangle_{j}$. We discuss two possible realizations of two qubit gates with this dynamics. The most straightforward way to implement a two-qubit gate is to just switch on the dipole-dipole interaction by exciting each qubit to the auxiliary state $|r\rangle$, conditioned on the initial logical state. This can be obtained by two resonant $\left(\delta_{1}=\delta_{2}=0\right)$ laser fields of the same intensity, corresponding to a Rabi frequency $\Omega_{1}=$ 
$\Omega_{2} \gg u$. After a time $\tau=\varphi / u$, the gate phase $\varphi$ is accumulated and the particles can be taken again to the initial internal state. However, besides $\varphi$ being sensitive to the atomic distance via the energy shift $u$, during the gate operation (i.e. when the the state $|r r\rangle$ is occupied) there are large mechanical effects, due to the dipole-dipole force, which create unwanted entanglement between the internal and the external degrees of freedom. These problems can be overcome by assuming single-qubit addressability and by moving to the opposite regime of small Rabi frequencies $\Omega_{1}(t) \neq \Omega_{2}(t) \ll u$. The gate operation is then performed in three steps, by applying: (i) a $\pi$-pulse to the first atom, (ii) a $2 \pi$-pulse (in terms of the unperturbed states) to the second atom, and, finally, (iii) a $\pi$-pulse to the first atom. The state $|00\rangle$ is not affected by the laser pulses. If the system is initially in one of the states $|01\rangle$ or $|10\rangle$ the pulse sequence (i)-(iii) will cause a sign change in the wave function. If the system is initially in the state $|11\rangle$ the first pulse will bring the system to the state $i|r 1\rangle$, the second pulse will be detuned from the state $|r r\rangle$ by the interaction strength $u$, and thus accumulate a small phase $\tilde{\varphi} \approx \pi \Omega_{2} / 2 u \ll \pi$. The third pulse returns the system to the state $e^{i(\pi-\tilde{\varphi})}|11\rangle$, which realizes a phase gate with $\varphi=\pi-\tilde{\varphi} \approx \pi$ (up to trivial single qubit phases). The time needed to perform the gate operation is of the order $\tau \approx 2 \pi / \Omega_{1}+2 \pi / \Omega_{2}$. Loss from the excited states $|r\rangle_{j}$ is small provided $\gamma \Delta t \ll 1$, i.e. $\Omega_{j} \gg \gamma$. A further improvement is possible by adopting chirped laser pulses with detunings $\delta_{1,2}(t) \equiv \delta(t)$ and adiabatic pulses $\Omega_{1,2}(t) \equiv \Omega(t)$, i.e., with a time variation slow on the time scale given by $\Omega$ and $\delta$ (but still larger than the trap oscillation frequency), so that the system adiabatically follows the dressed states of the Hamiltonian $H_{I}$. As found in [10, in this adiabatic scheme the gate phase is

$$
\varphi(\tau)=\int_{t_{0}}^{t_{0}+\tau} d t\left[\operatorname{sgn}(\tilde{\delta}) \frac{|\tilde{\delta}|-\sqrt{\tilde{\delta}^{2}+2 \Omega^{2}}}{2}-\operatorname{sgn}(\delta)\left(|\delta|-\sqrt{\delta^{2}+\Omega^{2}}\right)\right]
$$

with $\tilde{\delta}=\delta-\Omega^{2} /(4 \delta+2 u)$ the detuning including a Stark shift. For a specific choice of pulse duration and shape $\Omega(t)$ and $\delta(t)$ we achieve $\varphi(\tau)=\pi$. To satisfy the adiabatic condition, the gate operation time $\tau$ has to be approximately one order of magnitude longer than in the other scheme discussed above. In the ideal limit $\Omega_{j} \ll u$, the dipole-dipole interaction energy shifts the doubly excited state $|r r\rangle$ away from resonance. In such a "dipoleblockade" regime, this state is therefore never populated during gate operation. Hence, the mechanical effects due to atom-atom interaction are greatly suppressed. Furthermore, this version of the gate is only weakly sensitive to the exact distance between the atoms, since the distance-dependent part of the entanglement phase $\tilde{\varphi} \ll \pi$. For the same reason, possible excitations in the particles' motion do not alter significantly the gate phase, leading to a very weak temperature dependence of the fidelity.

In principle the controlled implementation of the above single and two- 
qubit quantum gates in the scalable setup of an optical lattice - avoiding any decoherence - allows any quantum computation to be performed, and in particular to run Shor's algorithm [31] for factoring large numbers in polynomial time. We note that the implementation of this factoring algorithm is one of the major practical goals in quantum computing as most current cryptographic systems rely on the difficulty of this task. However, for quantum computers to exceed classical ones in tasks like factoring they would have to operate thousands of qubits and the technology for achieving this goal might only be reachable in years from now. Still, there are also important nontrivial short term goals which can be realized with nowadays technology for quantum information processing. We present one of them in the following section.

\subsection{Feynman's universal quantum simulator}

A universal quantum simulator (QS) is a controlled device which efficiently reproduces the dynamics of any other many-particle system that evolves according to short range interactions. Therefore a QS could be used to efficiently simulate the dynamics of a generic many-body system, and in this way function as a fundamental tool for research in many body physics. Examples include spin systems, where classical simulations become nontrivial for a few tens of spins, and also interacting electrons moving in a magnetic field in 2D, which show interesting effects like the Fractional Quantum Hall effect. The simulation of such nontrivial systems is well suited for atoms trapped in an optical lattice and might therefore be implementable with resources available in the lab in the near future.

The realization of $2 \mathrm{D}$ motion in a $3 \mathrm{D}$ optical lattice is straightforward. Since the height of the barriers between sites adjacent in $z$-direction increases with the intensity of the corresponding pair of laser beams one can turn off any motion in $z$-direction by using high intensities for these lasers while keeping the barrier between sites adjacent in $x, y$ direction low. Atoms will then only be able to tunnel along the $x$ and $y$ direction and will thus be confined to one layer of the optical lattice. The physical simulation of a magnetic field in $z$ direction is less simple since time reversal symmetry needs to be broken. However, by accelerating the lattice and using additional laser beams as shown in 34 the simulation of a magnetic field can be achieved and allows to study 2D atom gases in effective magnetic fields. As was shown by Hofstaedter [33] for strong magnetic fields the energy bands form a fractal structure depending on the parameter $\alpha=A B e / 2 \pi \hbar$, where

$A$ is the area of one of the elementary cells of the lattice, $B$ is the strength of the magnetic field, and $e$ is the charge of the particles. Plotting the eigenenergies as a function of $\alpha$ yields the so called Hofstaedter butterfly shown in Fig. 10] While for electrons moving on a $2 \mathrm{D}$ surface it is very difficult to reach sufficiently large values of $\alpha$ to see this butterfly experimentally the 


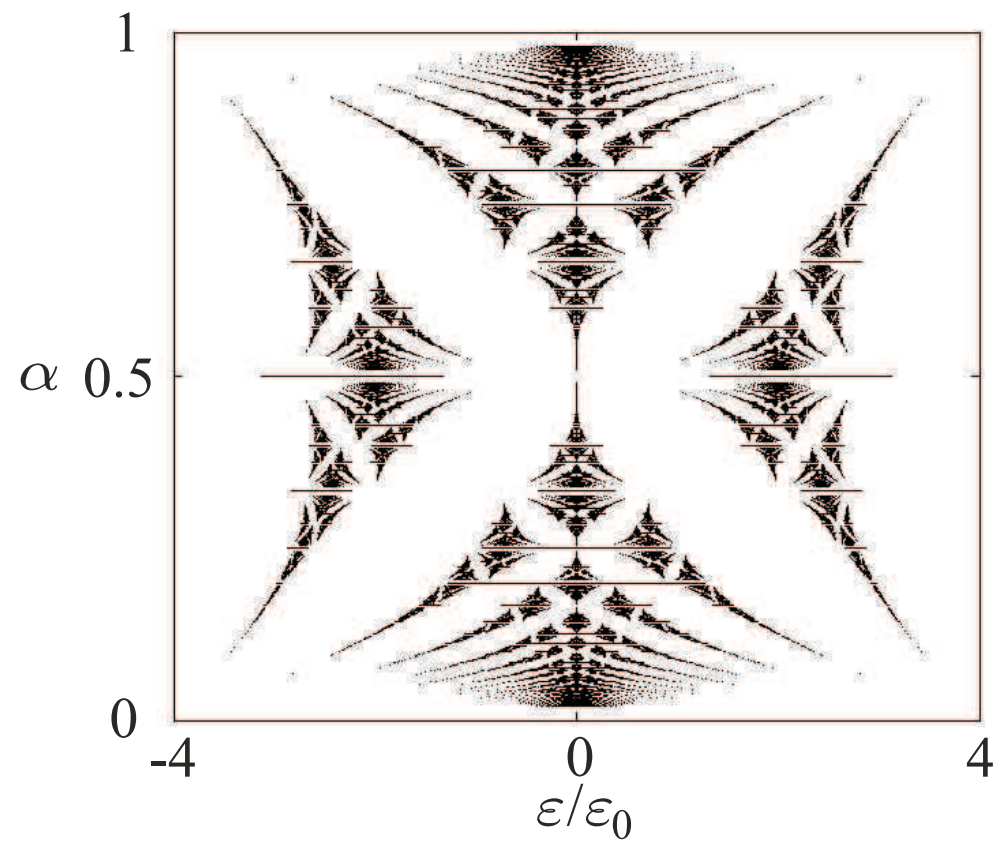

Figure 10: The Hofstaedter butterfly. Each dot in this fractal indicates an eigenenergy of the atoms moving in a magnetic field with parameter $\alpha$ as given in the text. The energy is normalized to $\epsilon_{0}$ which is given by the modulus of the hopping energy between two sites.

parameter $\alpha$ can be varied easily between 0 and 1 in an optical lattice and thus it should be possible to observe the energy band structures shown in Fig. 10.

The first idea for simulating (anti)ferromagnetic spin systems 32 in optical lattices came about after realizing that the interactions between atoms in optical lattices can be controlled [8, 9]. In a more general approach it was recognized by Jane et al. [1] that the nature of the controlled Hamiltonians available in optical lattices (and other quantum optical systems) is very well suited for simulating the time evolution of generic spin chains which are built up by qubits and evolve according to a Hamiltonian

$$
H_{N}=\sum_{\ell} H^{(\ell)}+\sum_{\ell \neq \jmath} H^{(\ell \jmath)}
$$

that decomposes into one-qubit terms $H^{(\ell)}$ and two-qubit terms $H^{\left(\ell_{\jmath}\right)}$. It can be shown that the time evolution resulting from $H_{N}$ is decomposable into a series of short pieces $K_{n}$ which are generated by single- and two-qubit evolutions. The single qubit evolution is realized in an optical lattice by properly shining a laser on the trapped atoms and two-qubit evolutions are obtained by operating two-qubit quantum gates via atom-atom interactions. 
A more detailed investigation shows that interesting spin-chain Hamiltonians like the (anti)ferromagnetic Heisenberg Hamiltonian can be simulated without the need for local addressing of single atoms and could thus be realized with standard optical lattice setups. The ability to perform independent operations on each of the qubits would allow all possible bipartite Hamiltonians to be simulated and in particular the study of quantum phase transitions in spin chains. We will next briefly study an optical lattice setup with Rydberg interactions between adjacent atoms which shows a quantum phase transition and could be viewed as a quantum simulator 27. We will also show how it can be used to implement an atom interferometer whose sensitivity is enhanced by the created entanglement. Furthermore, the ideas presented in the next section are the basis for implementing single qubit gates on robust qubits as introduced in Sec. 4.2.

\subsection{Multi particle maximally entangled states in optical lat- tices}

Let us assume that the Rydberg interactions between neighbouring atoms are turned on all the time and that the atoms are arranged in a 1D chain. Furthermore we allow each atom to either tunnel between two adjacent wells whose ground states are denoted by $|a\rangle$ and $|b\rangle$ or to have an additional laser driving Raman transitions between two internal states (again denoted by $|a\rangle$ and $|b\rangle)$. This situation is schematically shown in Fig. 11

The dynamics of this setup can be understood as follows. Hopping between the two modes $|a\rangle_{j}$ and $|b\rangle_{j}$ of the $j$-th atom is described by

$$
H_{h}=B \sum_{j}\left(|a\rangle_{j}\langle b|+\text { h.c. }\right) \equiv B \sum_{j} \sigma_{x}^{(j)}
$$

with $\sigma_{x}^{(j)}$ the Pauli $x$-matrix for the two states (viewed as a spin) of the $j$-th atom and $B>0$ the energy associated with this process. The ground state of this Hamiltonian is obtained by putting each atom into an equal superposition of states written in spin notation as $\left|\downarrow_{x}\right\rangle_{j}=\left(\left|\uparrow_{z}\right\rangle-\left|\downarrow_{z}\right\rangle\right) / \sqrt{2}$, where $\left|\uparrow_{z}\right\rangle \equiv|a\rangle$ and $\left|\downarrow_{z}\right\rangle \equiv|b\rangle$ i.e. we find

$$
\left|\Psi_{h}\right\rangle=\left|\downarrow_{x} \downarrow_{x} \downarrow_{x} \cdots \downarrow_{x}\right\rangle
$$

Note that this part of the Hamiltonian is identical to a spin chain in a magnetic field $B$ along the $x$-axis.

The interaction between two nearest neighbours due to Rydberg interactions is given by

$$
H_{i}=W \sum_{j} \sigma_{z}^{(j)} \sigma_{z}^{(j+1)}
$$

accounting for the energy difference of having two adjacent particles in the same vs. different internal states. In this case the ground state depends on 


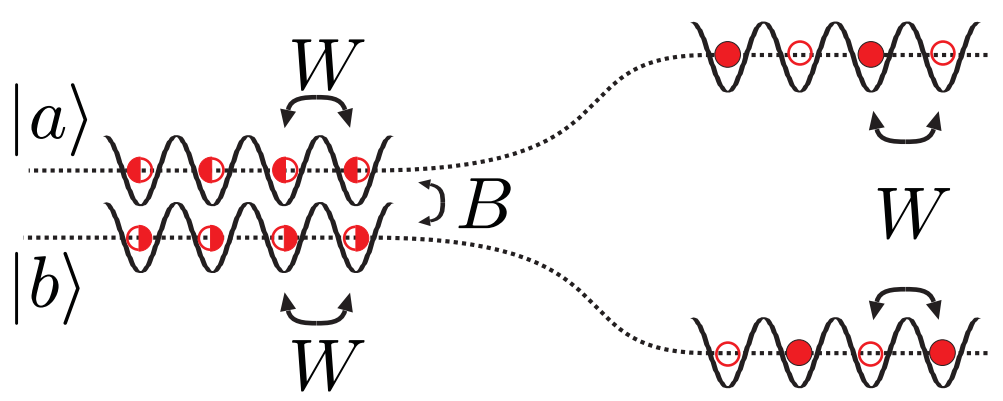

Figure 11: Creation of robust multi particle entangled states in 1D beam splitter setups. Closed circles indicate an atom open circles an empty position.

the sign of the interaction parameter $W$. For repulsive interactions $W>0$ the interaction will be minimized by arranging the particles as

$$
\left|\psi_{i r}\right\rangle=\alpha\left|\uparrow_{z} \downarrow_{z} \cdots \uparrow_{z} \downarrow_{z}\right\rangle+\beta\left|\downarrow_{z} \uparrow_{z} \cdots \downarrow_{z} \uparrow_{z}\right\rangle
$$

while for attractive interactions the ground state is

$$
\left|\psi_{i a}\right\rangle=\alpha\left|\uparrow_{z} \uparrow_{z} \cdots \uparrow_{z}\right\rangle+\beta\left|\downarrow_{z} \downarrow_{z} \cdots \downarrow_{z}\right\rangle
$$

Both of these states are maximally entangled multi particle states, i.e. extensions of the well known GHZ states for three particles.

In the total Hamiltonian $H=H_{h}+H_{i}$ the interaction energy and the hopping energy compete with each other resulting in a quantum phase transition. When the interaction energy is kept constant and the hopping term is switched off adiabatically, as e.g. shown in Fig. 11 the state of the system will dynamically change from $\left|\Psi_{h}\right\rangle$ which is a product state to one of the two states $\left|\Psi_{i r}\right\rangle$ or $\left|\Psi_{i a}\right\rangle$ depending on the sign of the interaction. The exact values of the parameters $\alpha$ and $\beta$ depend on the details of the dynamics and are discussed in [27. These maximally entangled states can serve different purposes depending on the sign of $W$. Let us discuss the possible applications of these two kinds of maximally entangled states

\subsection{Repulsive interactions}

In this case both parts of the superposition state have the same number of particles in each of the two internal states and thus external stray fields act identically on both, therefore not affecting the parameters $\alpha$ and $\beta$. Because of this stability one can use them for storing quantum information in a robust way as described in Sec. 4.2. Single qubit gates can be performed by dynamically going back and forth through the quantum phase transition in the whole chain changing the parameters $\alpha$ and $\beta$ in a controlled way. 


\subsection{Attractive interactions}

For attractive interactions the terms in the superposition of $\left|\psi_{i a}\right\rangle$ will respond to external fields very differently. Therefore the relative phase between the parameters $\alpha$ and $\beta$ will be very susceptible to these fields, in fact for $N$ particles in the chain this phase will be $N$ times larger than if there was just a single particle. The two parts of $\left|\psi_{i a}\right\rangle$ can thus be used as two arms of an entanglement enhanced atomic interferometer.

\section{Summary}

This article reviewed recent advances in controlling and manipulating neutral atoms by quantum optical methods concentrating on the use of optical lattices. We investigated novel physical features arising from interactions in ultracold dense clouds of atoms as provided by BEC and showed how they enable experimentalists to gain control over the spatial degrees of freedom of individual atoms. We also studied possible new applications - in particular in quantum information processing - which make use of this unprecedented quantum control. We showed that universal sets of quantum gates can be implemented and how they might lead to a universal scalable quantum computer in the future. Furthermore we also investigated possibilities for shorter term applications of optical lattices as quantum simulators for strongly correlated systems.

\section{References}

[1] M.H. Anderson, J.R. Ensher, M.R. Matthews, C.E. Wieman, and E.A. Cornell, Science, 269, 198 (1995); K.B. Davies, M.-O. Mewes, M.R. Andrews, N.J. van Druten, D.S. Durfee, D.M. Kurn, and W. Ketterle, Phys. Rev. Lett. 75, 3969 (1995); C.C. Bradley, C.A. Sackett, J.J. Tollett, and R.G. Hulet, Phys. Rev. Lett. 75, 1687 (1995).

[2] For a review see, e.g.: J.R. Anglin and W. Ketterle, Nautre 416, 211 (2002); F. Dalfovo S. Giorgini, L.P. Pitaevskii, and S. Stringari, Rev. Mod. Phys. 71, 463 (1999); L.P. Pitaevskii and S. Stringari, BoseEinstein condensation, Oxford University Press (2003).

[3] For further information see: http://www.nobel.se/physics/laureates/2001/

[4] B.P. Anderson, and M.A. Kasevich, Science 282, 1686 (1998); C. Orzel, A.K. Tuchman, M.L. Fenselau, M. Yasuda, and M.A. Kasevich, Science, 291, 2386 (2001). 
[5] W.K. Hensinger, H. Häffner, A. Browaeys, N.R. Heckenberg, K. Helmerson, C. McKenzie, G.J. Milburn, W.D. Phillips, S.L. Rolston, H. Rubinsztein-Dunlop, and B. Upcroft, Nature • 412, 52 (2001).

[6] M. Greiner, O. Mandel, T. Esslinger, T.W. Haensch, I. Bloch, Nature 415, 39 (2002).

[7] R. Wynar et al., Science 287, 1016 (2000); J. Javanainen and M. Mackie, Phys. Rev. Lett. 88, 90403 (2002); D. Jaksch, V. Venturi, J. I. Cirac, C. J. Williams, and P. Zoller, Phys. Rev. Lett. 89, 040402 (2002).

[8] D. Jaksch, H.-J. Briegel, J. I. Cirac, C. W. Gardiner, and P. Zoller, Phys. Rev. Lett. 82, 1975 (1999).

[9] G.K. Brennen, C.M. Caves, P.S. Jessen, and I.H. Deutsch, Phys. Rev. Lett. 82, 1060 (1999).

[10] D. Jaksch, J.I. Cirac, P. Zoller, S.L. Rolston, R. Cote, and M.D. Lukin, Phys. Rev. Lett. 85, 2208 (2000).

[11] E. Jane, G. Vidal, W. Dür, P. Zoller, J.I. Cirac, Quantum Information and Computation, 3, 15 (2003).

[12] J.K. Pachos and P.L. Knight, Phys. Rev. Lett. 91, 107902 (2003).

[13] J. Mompart, K. Eckert, W. Ertmer, G. Birkl, M. Lewenstein, Phys. Rev. Lett. 90, 147901 (2003).

[14] T. Pellizzari, S.A. Gardiner, J.I. Cirac, and P. Zoller, Phys. Rev. Lett. 75, 3788 (1995).

[15] Q.A. Turchette, C.S. Wood, B.E. King, C.J. Myatt, D. Leibfried, W.M. Itano, C. Monroe, and D.J. Wineland, Phys. Rev. Lett. 81, 3631 (1998); D. Leibfried, B. DeMarco, V. Meyer, D. Lucas, M. Barrett, J. Britton, W.M. Itano, B. Jelenkovi, C. Langer, T. Rosenband, and D.J. Wineland, Nature 422, 412 (2003).

[16] D. Kielpinski, C. Monroe, and D.J. Wineland, Nature 417, 709 (2002).

[17] S. Gulde, M. Riebe, G.P.T. Lancaster, C. Becher, J. Eschner, H. Häffner, F. Schmidt-Kaler, I.L. Chuang, and R. Blatt, Nature 421, 48 (2003); F. Schmidt-Kaler, H. Häffner, M. Riebe, S. Gulde, G.P.T. Lancaster, T. Deuschle, C. Becher, C.F. Roos, J. Eschner, and R. Blatt, Nature 422, 408 (2003).

[18] L.M.K. Vandersypen, M. Steffen, G. Breyta, C.S. Yannoni, M.H. Sherwood, and I.L. Chuang, Nature 414, 883 (2001); H.K. Cummins, 
C. Jones, A. Furze, N.F. Soffe, M. Mosca, J.M. Peach, and J.A. Jones, Phys. Rev. Lett. 88, 187901 (2002).

[19] Y. Yu, S. Han, X. Chu, S.-I. Chu, and Z. Wang, Science 296, 889 (2002); J. Martinis, S. Nam, J. Aumentado, and C. Urbina, Phys. Rev. Lett. 89, 117901 (2002); Yu.A. Pashkin, T. Yamamoto, O. Astafiev, Y. Nakamura, D.V. Averin, and J.S. Tsai, Nature 421, 823 (2003); T. Yamamoto, Yu.A. Pashkin, O. Astafiev, Y. Nakamura, and J.S. Tsai, Nature 425, 941 (2003).

[20] X. Li, Y. Wu, D. Steel, D. Gammon, T.H. Stievater, D.S. Katzer, D. Park, C. Piermarocchi, and L.J. Sham, Science 301, 809 (2003); B.E. Kane, Nature 393, 133 (1998).

[21] A.J. Ferguson, P.A. Cain, D.A. Williams, and G.A.D. Briggs, Phys. Rev. A 65, 034303 (2002).

[22] S. Friebel, C. D'Andrea, J. Walz, M. Weitz, and T.W. Hänsch, Phys. Rev. A 57, R20 (1998).

[23] D. Jaksch, C. Bruder, J.I. Cirac, C.W. Gardiner, P. Zoller, Phys. Rev. Lett. 81, 3108 (1998).

[24] D. Deutsch, Proc. Roy. Soc. Lond. A, 425, 73 (1989); D.P. DiVincenzo, Phys. Rev. A 50, 1015 (1995); H.F. Chau and F. Wilczek, Phys. Rev. Lett. 75, 748 (1995); A. Barenco, Proc. Roy. Soc. Lond. A 449, 679 (1995); D. Deutsch, A. Barenco and A. Ekert, Proc. Roy. Soc. Lond. A 449, 669 (1995).

[25] D.P. DiVincenzo, Fortschr. Phys. 48, 771 (2000).

[26] O. Mandel et al., Nature 425, 937 (2003).

[27] U. Dorner, P. Fedichev, D. Jaksch, M. Lewenstein, and P. Zoller, Phys. Rev. Lett. 91, 073601 (2003).

[28] L.-M. Duan, J.I. Cirac, and P. Zoller, Science 292, 1695 (2001).

[29] T. Calarco, H.-J. Briegel, D. Jaksch, J.I. Cirac and P. Zoller, J. Mod. Opt. 47, 2137 (2000).

[30] S. Peil, J.V. Porto, B. Laburthe Tolra, J.M. Obrecht, B.E. King, M. Subbotin, S.L. Rolston, and W.D. Phillips, Phys. Rev. A 67, 051603(R) (2003).

[31] P. Shor, Proc. 35th An. Symp. On Foundations of Comp. Sci., IEEE Press, Los Alamitos, CA, 1994.

[32] A. Sørensen and Klaus Mølmer, Phys. Rev. Lett. 83, 2274 (1999). 
[33] D.R. Hofstadter, Phys. Rev. B 14, 2239 (1976).

[34] D. Jaksch and P. Zoller, NJP 5, 56 (2003).

Dr. Dieter Jaksch obtained his PhD from the University of Innsbruck in 1999. He moved to the University of Oxford where he currently holds a lecturer position at the Clarendon Laboratory in the Department of Physics in 2003. Dieter Jaksch started to work on ultracold gases in optical lattices in 1997, his current interests are mainly in quantum computing applications of quantum optical systems. 